\title{
LA REFUTACIÓN DE LA TEORÍA CUANTITATIVA DEL DINERO
}

\author{
JUAN RAMÓN RALLO*
}

En las primeras páginas de su The Theory of Prices, Arthur Marget insiste en que los economistas tienden a confundir la ecuación cuantitativa $-\mathrm{M}^{*} \mathrm{~V}=\mathrm{P} * \mathrm{Q}-$ con la teoría cuantitativa del dinero (Marget 1938, 9-39). Si bien, como asimismo sostiene Benjamin Anderson (Anderson 1917, 161), la ecuación cuantitativa es en realidad una identidad contable irrefutable que tan sólo pone de manifiesto que el conjunto de los pagos realizados en una economía $-\mathrm{M}^{*} \mathrm{~V}$ - es igual al conjunto de los cobros $-\mathrm{P}^{*} \mathrm{Q}-$, la teoría cuantitativa asume la existencia de unas relaciones concretas entre las cuatro variables de la ecuación cuantitativa.

Aunque, como sucede con la teoría del capital, existen casi tantas teorías cuantitativas como economistas, Joseph Schumpeter, en su monumental Historia del análisis económico, tasaba en cuatro las proposiciones básicas que se encuentran presentes en cualquier teoría cuantitativa: a) la oferta monetaria $(\mathrm{M})$ es una variable independiente de los precios $(\mathrm{P})$ y del volumen de transacciones $(\mathrm{Q})$; b) la velocidad del dinero (V) está determinada por factores institucionales y, o bien no varía, o bien varía muy lentamente; c) la cantidad de transacciones $(Q)$ no depende de la oferta monetaria (M); y d) los cambios en la oferta monetaria (en M), a menos que vayan casualmente de la mano de cambios en la cantidad de transacciones (en $\mathrm{Q}$ ), provocan variaciones mecanicistas en todos los precios de la economía, con independencia de cómo se haya producido esa variación en la oferta monetaria (Schumpeter 1954, 703).

En efecto, si algo pretende probar la teoría cuantitativa es que, invariadas las restantes circunstancias, un incremento de $\mathrm{M}$

\footnotetext{
* Doctor en Economía. Profesor del Departamento de Economía Aplicada I de la Universidad Rey Juan Carlos y del centro de estudios ISEAD.
} 
provocará un aumento proporcional de $\mathrm{P}$, y para ello resulta necesario asumir que $M$ es independiente de $V$ y de $Q$ (y $V$ y $Q$ de $\mathrm{M}$ ) y que el aumento de $\mathrm{P}$ es una consecuencia de $\mathrm{M}$ y no al revés.

Pese a la popularidad lograda por la teoría cuantitativa, las cuatro hipótesis de partida son falsas. No quiero con ello negar que, en general, los aumentos de $\mathrm{M}$ no se traduzcan en ciertos incrementos de $\mathrm{P}$ - como decía Hayek, «desde un punto de vista práctico, pocas cosas podrían ser más graves que el público dejara de creer en las proposiciones básicas de la teoría cuantitativa» (Hayek 1967, 3-4) - , pero en aras del rigor teórico es inadmisible que aceptemos a pies juntillas unos razonamientos muy de brocha gorda que nos ocultan las auténticas interrelaciones entre el dinero, el crédito y los bienes económicos. Por ello, en este artículo me centraré en analizar y refutar cada una de las cuatro hipótesis básicas de la teoría cuantitativa.

\section{I \\ LA OFERTA MONETARIA ES UNA VARIABLE INDEPENDIENTE DE LOS PRECIOS}

La primera de ellas es que $M$ es una variable independiente de $P$ y de $Q$; en otras palabras, la oferta monetaria es reputada como una variable exógena al sistema que no reacciona ni ante las variaciones del conjunto de precios ni del conjunto de transacciones económicas.

Tal como sucederá con respecto al resto de variables, en este caso hay un claro problema inicial de indeterminación: ¿qué entendemos por $\mathrm{M}$, esto es, por la oferta monetaria? En términos estrictos, podríamos entender por oferta monetaria la cantidad de dinero en una sociedad, a saber, el conjunto de los bienes presentes con una elevada liquidez (por ejemplo, el oro, la plata...), lo que la economía neoclásica llamaría la base monetaria o M0; esto excluye de la oferta monetaria a los derechos de cobro, que no son dinero sino derechos a recibir dinero (por ejemplo, los depósitos bancarios). Con estos parámetros, la hipótesis de la teoría cuantitativa sería sólo levemente incorrecta: la cantidad de bienes 
presentes líquidos nos vendría dada y sería bastante independiente de P y Q. Sólo deberíamos adaptar la hipótesis para contemplar que la reducción de $\mathrm{P}$ (de los precios de la economía) supone un incremento del poder adquisitivo de $\mathrm{M}$, lo que por lo general se traducirá en un incentivo a incrementar la oferta de dinero (si el poder adquisitivo del oro aumenta, la producción minera de oro también subirá).

El problema es que si sólo incluimos la cantidad de dinero en $\mathrm{M}$, el resto de corolarios de la teoría cuantitativa se caen por su propio peso. Al cabo, es evidente que sobre los precios - al menos a corto y medio plazo - no sólo influye la cantidad de dinero, sino también la cantidad (y calidad) de promesas a entregar dinero. Por consiguiente, hemos de flexibilizar lo suficiente $\mathrm{M}$ como para incluir todas aquellas promesas que se empleen como medios de pago. Pero en ese caso, la hipótesis de que $\mathrm{M}$ es independiente de $\mathrm{P}$ y $\mathrm{Q}$ deja de ser cierta.

En efecto, uno de los factores que determina el volumen de las promesas de pago de una economía es la cantidad y el precio de los bienes que pueden emplearse como colateral de esas promesas de pago. Tomemos el caso de una letra de cambio: un incremento en la cantidad o en el precio de los bienes de consumo altamente demandados permite girar nuevas letras de cambio, aumentando de ese modo la cantidad de promesas de pago y la «oferta monetaria» (M) en sentido lato. Análogamente, un incremento de la cantidad y del precio de los inmuebles permite a los bancos dispuestos a degradar su liquidez concediendo hipotecas más cuantiosas, las cuales se traducen en una mayor cantidad de depósitos a la vista para los hipotecados y, por consiguiente, en un incremento de $\mathrm{M}$.

En definitiva, la teoría cuantitativa ignora que la «oferta monetaria» es un resultado endógeno del proceso económico y no un dato exógeno que nos viene dado. Su obsesión teórica es unidireccional $-\mathrm{M}$ empuja al alza a $\mathrm{P}-$, olvidándose de los efectos boomerang que existen - P empuja al alza a $\mathrm{M}$-.

Una teoría cuantitativa más realista, blindada contra estos errores de fondo, debería considerar que $\mathrm{M}$ se divide en dos partes: el dinero y las promesas de entregar dinero (en ciertas formulaciones de la teoría cuantitativa sí aparecen diferenciadas, nosotros 
las llamaremos $\mathrm{M}^{\prime}$ y $\mathrm{M}^{\prime \prime}$ respectivamente). A su vez, debería tener en cuenta que el dinero $\left(\mathrm{M}^{\prime}\right)$ es en realidad un subconjunto variable de $\mathrm{Q}$ (aquella porción de los bienes presentes que sean además líquidos), determinado por los procesos de monetización y desmonetización (cambios en la demanda monetaria) de unos bienes económicos que a su vez pueden fabricarse atendiendo a los cambios en la rentabilidad de producirlos. Y asimismo, habría que tener en cuenta que las promesas a entregar dinero $\left(\mathrm{M}^{\prime \prime}\right)$ estarán compuestas en parte por un subconjunto de $\mathrm{M}^{\prime}$ (los bienes presentes líquidos que se utilicen como garantía o respaldo de las promesas de pago) y en parte, si los agentes no se preocupan por su liquidez, por otro conjunto tremendamente fluctuante como es la oferta de bienes futuros ( $Q$ futuro), que se empleará como inadecuada garantía para las promesas de pago presentes.

Por eso mismo, además, no sólo será relevante estudiar la cantidad de $\mathrm{M}$, sino también su calidad: dado que la oferta de bienes futuros es muy incierta y está muy sometida a las oscilantes expectativas de los agentes, un incremento del valor presente de esos bienes futuros (por ejemplo, vía descensos de los tipos de interés) o una mejora generalizada de las expectativas (propia de auges y burbujas) generará emisiones masivas de promesas de pago contra bienes futuros (aumentos de $\mathrm{M}^{\prime \prime}$ ) que, debido a la euforia irracional, serán aceptadas por todos los agentes a su importe nominal; en cambio, reducciones del valor presente de los bienes futuros o empeoramientos generalizados de las expectativas limitarán su emisión y su aceptación (hasta el punto de que muchas de las promesas podrían dejar de circular salvo a grandes descuentos, lo que les restaría casi cualquier influencia sobre P).

En todo caso, si esta primera hipótesis de la teoría cuantitativa (que la oferta monetaria es independiente del nivel de precios) se releja lo suficiente como para volverla realista, es evidente que la teoría cuantitativa queda seriamente tocada: si una variación autónoma de $\mathrm{P}$ puede generar una variación de $\mathrm{M}$, habrá que reconocer que existen otras causas para que se produzcan variaciones de $\mathrm{P}$ más allá de los cambios en $\mathrm{M}$; y si, como decimos, un incremento de $Q$ puede dar lugar a un aumento de $M$, también resultará evidente que no todo aumento de $\mathrm{M}$ generará un alza 
de $\mathrm{P}$ (si el aumento de $\mathrm{Q}$ genera un aumento de $\mathrm{M}, \mathrm{P}$ podrá mantenerse estable). En otras palabras, la teoría cuantitativa tradicional deja de ser una teoría general capaz de explicar todas las variaciones del conjunto de precios de una economía y pasa a ser una teoría particular aplicable sólo a unas condiciones determinadas y bajo unas restricciones muy concretas.

II

\section{LA VELOCIDAD DEL DINERO ESTÁ DETERMINADA POR FACTORES INSTITUCIONALES Y APENAS VARÍA}

La segunda hipótesis en la que se sustenta la teoría cuantitativa es que la velocidad de circulación del dinero (V) es constante e independiente del resto de variables, quedando determinada por factores institucionales. Que la velocidad sea independiente es un requisito teórico para poder relacionar las variaciones de la cantidad de dinero (M) con las de los precios (P); que sea constante es una conveniencia empírica que ahorra trabajo a los economistas a la hora de recolectar datos y «demostrar» la fuerte relación entre oferta monetaria y precios. Como en las restantes relaciones entre variables, en este caso parece que se parte de una conclusión preconcebida (la teoría cuantitativa es cierta) para, a partir de ella, desplegar las hipótesis necesarias que la hagan verosímil; pero, como en los otros casos, constituye un error económico mayúsculo.

Recordemos que la velocidad del dinero no es más que la inversa de la demanda de dinero (esto es, la inversa del tiempo medio que los agentes atesoran una determinada cantidad de dinero). Por tanto, en lugar de pensar en términos de velocidad - las más de las veces un concepto poco intuitivo- podemos simplemente razonar en términos de atesoramiento de dinero.

Para empezar, debemos reconocer que el atesoramiento sí está en parte determinado por factores institucionales y consuetudinarios. Cuanto más desarrollado se encuentre el sistema de pagos de un país y cuanto menos habituados estén los agentes a abonar sus operaciones en efectivo, menos tiempo permanecerá cada unidad monetaria dentro de los saldos de caja de un 
individuo. Así, por ejemplo, una economía muy bancarizada donde el medio preferente de pago sean los cheques o las tarjetas de débito, lo habitual será que se produzcan un número de transacciones mucho mayor que en una economía donde todos los pagos se produzcan al contado y que, en consecuencia, la velocidad de circulación del dinero también sea mayor.

Ahora bien, que la velocidad del dinero esté en parte determinada por factores institucionales no significa ni que esté sólo determinada por factores institucionales, ni que esté mayormente determinada por factores institucionales, ni que sea independiente del resto de variables de la ecuación cuantitativa. Al contrario de lo que los cuantitativistas se ven forzados a suponer para no abandonar su insostenible teoría, el resto de variables sí ejercen una enorme influencia sobre la misma.

Empecemos por el lado derecho de la ecuación: el precio y la cantidad de bienes $\left(\mathrm{P}^{*} \mathrm{Q}\right)$. Hasta cierto punto, el atesoramiento presente viene determinado por el poder adquisitivo futuro del dinero (por cuántos bienes podrán adquirirse con una unidad monetaria): si el agente espera que los precios futuros caigan, incrementará su atesoramiento - esto es, se pondrá largo en dinero y corto en mercancías-; si, en cambio, espera que los precios futuros aumenten, reducirá su atesoramiento -esto es, se pondrá corto en dinero y largo en mercancías-.

No se trata, por supuesto, de una relación ni absolutamente rígida ni tenebrosamente apocalíptica como muchas veces nos quieren hacer creer los keynesianos a cuenta de Japón («nadie consume porque todos esperan que caigan los precios»). Primero, porque el mero acto de atesorar o desatesorar dinero adelanta la caída o el aumento de precios esperado, de modo que la tendencia a atesorar o desatesorar dinero sólo continuará si las expectativas bajistas o alcistas se mantienen. Segundo, porque otra forma de ponerse largos en dinero futuro es invirtiendo el dinero presente en proyectos que venzan en el momento en que se desea disponer del dinero. $Y$ tercero, porque aun cuando el dinero permaneciera atesorado, se desatarían otras fuerzas - como la caída del tipo de descuento- que permitirían reorientar la estructura productiva de la economía sin irresolubles quebrantos. Pero, por poco rígida o apocalíptica que sea la relación entre 
la velocidad del dinero y su poder adquisitivo, es indudable que existe y que debe ser tenida en cuenta.

Más significativa, con todo, es la relación entre M y V. Fijémonos en que ambas variables son necesarias para explicar cuánto dinero se está utilizando con el propósito de adquirir bienes $(Q)$ : $\mathrm{M}$ es la oferta monetaria, entendida como todo el stock de medios de pago (dinero y promesas de pago), y $\mathrm{V}$ es la inversa de la demanda monetaria, entendida como la parte del stock de dinero que se está utilizando para efectuar pagos. V nos informa de qué múltiplo de los medios de pago se destinan en un momento dado para alcanzar bienes (o, si tomamos la inversa, es decir, la demanda de dinero, qué porcentaje de los medios de pago se atesoran). Parece claro, pues, que difícilmente podrán estas dos variables ser independientes cuando $\mathrm{V}$ necesita nutrirse de $\mathrm{M}$, esto es, cuando la constitución de los saldos deseados de tesorería se realizará a partir de una oferta monetaria determinada. Así, por ejemplo, si en ciertos contextos como el actual los agentes atesoran todos los medios de pago que el banco central pone a su disposición - esto es, si deciden no hacer uso de los medios de pago que el banco central pone a su disposición-, M aumentará a la vez que $V$ disminuirá: habrá más oferta de dinero pero ésta será completamente reabsorbida por la demanda. El cuantitativismo es demasiado simple al presuponer que todo aumento en la cantidad de dinero dará lugar a un incremento proporcional en la circulación de dinero: en ocasiones un aumento de M generará un incremento más que proporcional de $\mathrm{V}$ (inflaciones o hiperinflaciones) y en otras una reducción (deflaciones).

De este modo, por visualizar las relaciones entre $\mathrm{M} \mathrm{y} \mathrm{V}$ en un horizonte temporal más prolongado, tengamos en cuenta que cuando aumenta la oferta monetaria merced a la emisión de promesas de pago $\left(\mathrm{M}^{\prime \prime}\right)$ garantizadas por bienes de consumo futuros ( $Q$ futuros), el nuevo crédito presionará al alza los precios de todos los bienes presentes $(\mathrm{P})$, lo que incentivará por los motivos ya apuntados un desatesoramiento de dinero (aumento de V) que hasta cierto punto se realimentará con el aumento esperado de precios. Conforme $\mathrm{M}^{\prime \prime}$ siga aumentando, llegará un momento en el que los agentes comenzarán a darse cuenta de que la calidad de esas promesas de pago se ha ido deteriorando en la medida 
en que no pueden satisfacerse todas (los bienes necesarios para saldarlas no existen en el presente), por lo que procederán a ponerse cortos en las promesas de pago $\left(\mathrm{M}^{\prime \prime}\right)$ y largos en dinero $\left(\mathrm{M}^{\prime}\right)$; es decir, comenzarán a liquidar las promesas de pago de peor calidad y a atesorar dinero (reducción de V). Este atesoramiento de dinero unido a la hipertrofia de las promesas de entregar dinero generará una cadena de impagos en estas últimas (contracción de $\mathrm{M}^{\prime \prime}$ ), lo que a su vez dará lugar a una caída de precios (reducción de P) y ésta, a su vez, reforzará el atesoramiento de dinero (reducción V) hasta que la calidad de las promesas de pago se estabilice y los precios relativos de los bienes se hayan ajustado a la baja.

Vemos, por consiguiente, que lejos de ser variables independientes, $\mathrm{M}, \mathrm{P}$ y $\mathrm{Q}$ influyen decisivamente sobre $\mathrm{V}, \mathrm{y} \mathrm{V}$ influye decisivamente sobre $\mathrm{M}, \mathrm{P}$ y $\mathrm{Q}$. El problema es que relajar la hipótesis de esta independencia socavaría la teoría cuantitativa: si un aumento de $\mathrm{M}$ puede dar lugar a una caída a corto o a largo plazo de $\mathrm{V}$, o si un aumento de $\mathrm{P}$ puede realimentarse con aumentos de $\mathrm{V}$, parece claro que no podrá establecerse una relación unívoca entre $\mathrm{P}$ y M: M podrá aumentar sin que lo haga P y P podrá aumentar sin que lo haga $\mathrm{M}$.

III

\section{LA CANTIDAD DE TRANSACCIONES NO DEPENDE DE LA OFERTA MONETARIA}

La tercera de las hipótesis sobre la que se sustenta la teoría cuantitativa del dinero es que la cantidad de transacciones $(Q)$ no se ve influida por la oferta monetaria (M). Se trata de un requisito esencial para validar la tesis final de que el aumento de $\mathrm{M}$ engendra un incremento proporcional de P: al fin y al cabo, si las variaciones de $\mathrm{M}$ pudieran trasladarse total o parcialmente a variaciones de $Q$ - como sí sería factible desde un punto de vista matemático, pues recordemos que $\mathrm{M}^{*} \mathrm{~V}=\mathrm{P}^{*} \mathrm{Q}$-, la teoría cuantitativa se vería reducida a cenizas.

Ahora bien, ¿cuán realista resulta esta hipótesis? En principio parece que bastante: por el hecho de aumentar la cantidad de 
medios de pago, el número de bienes y servicios no se incrementa. Por consiguiente, el único resultado que debería de tener el crecimiento de $\mathrm{M}$ es un alza de $\mathrm{P}$.

Sin embargo, ya hemos apuntado a una de las razones por las que, siendo rigurosos, esta conclusión resulta engañosa: si M depende en parte de $Q$, es cuando menos dudoso que manteniendo constante $\mathrm{M}$ algunos incrementos de $\mathrm{Q}$ pudieran llegar a darse. No estoy diciendo, claro, que la cantidad de bienes y servicios no pueda crecer mediante reducciones de los precios (deflación), ni siquiera que resulte conveniente que todo aumento de $Q$ sea esterilizado nominalmente por un incremento de $M$, sino que de la misma manera que la aparición del dinero mejora la coordinación entre seres humanos (y por tanto incrementa el número de transacciones económicas) con respecto al trueque, en ciertos contextos la creación privada de medios de pago adicionales puede mejorar la coordinación entre los agentes y, por consiguiente, incrementar Q. Por ejemplo, es muy probable que sin el recurso a las letras de cambio las ferias medievales nunca se hubieran organizado, ya que la mayoría de mercaderes, si bien disponía de mercancías para ofertar, carecía de oro con el que pagar las que quería adquirir (por no hablar del elevado riesgo que suponía transportar el oro desde el punto de origen hasta la feria). La emisión de letras de cambio respaldadas por sus mercancías líquidas les permitió a los mercaderes superar este inconveniente, de modo que las ferias, y las transacciones, pudieron perfeccionarse.

Pero además, existen otras razones para poner en duda la ausencia de relación entre la cantidad de medios de pago y el volumen de operaciones comerciales.

Primero, los períodos de rápido aumento o de rápida contracción de los medios de pago (asociados con hiperinflaciones e hiperdeflaciones) van asociados con retenciones de activos y mercancías fuera del mercado o con liquidaciones de activos y mercancías. En concreto, durante las hiperinflaciones los agentes retienen sus activos y mercancías (incluso para el autoconsumo), pues el envilecimiento del dinero puede llegar a ser tan rápido que las ventas resultan del todo ruinosas; en cambio, durante las hiperdeflaciones - generales o concentradas en sectores concretos- sucede 
justo lo contrario: el valor del dinero se incrementa tan rápido frente al de los activos y las mercancías que todo el mundo está deseoso de enajenarlos y atesorar oro. Además, este último proceso se verá generalmente acompañado por impagos de deuda y liquidaciones forzosas, las cuales provocarán un incremento coactivo de los activos y las mercancías disponibles para la venta (cierto es que también puede generarse el efecto contrario: si durante una contracción del crédito se incrementa la cantidad de medios de pago para refinanciar las deudas vencidas, el número de liquidaciones será menor del que debiera ser y por tanto no se ofertará tanta $Q$ como debería). En cualquiera de los dos casos, pues, las variaciones de $\mathrm{M}$ generan variaciones reales en las transacciones efectuadas (en el número de compraventas); ya sea para minorarlo o para acelerarlo.

Segundo, como también hemos dicho, las variaciones menos bruscas de precios (inflaciones y deflaciones moderadas, derivadas por ejemplo de la producción de oro o del incremento de la cantidad de bienes y servicios) pueden realimentarse con el atesoramiento o desatesoramiento de dinero (reducciones o aumentos de $\mathrm{V}$, respectivamente) y el desatesoramiento puede dar lugar a incrementos sostenibles, a corto plazo, de la producción. Al desatesorarse el dinero - reservas de poder adquisitivo de los agentes- las empresas y los factores productivos pasan a operar de manera transitoria a un ritmo superior a aquel al que venían operando: a cambio de remuneraciones extraordinarias, los empresarios exponen a su maquinaria a un mayor desgaste y los trabajadores aceptan alargar sus jornadas laborales, redundando todo ello en un aumento acelerado - y transitorio- de Q. Por consiguiente, un aumento de $\mathrm{M}$ (producción de oro) puede generar un aumento de $\mathrm{V}$ (desatesoramiento de dinero ante la perspectiva de alzas en los precios) y un aumento de $\mathrm{V}$ puede provocar una expansión de $\mathrm{Q}$.

Y, por último, la variación de los medios de pago también puede dar lugar a aumentos insostenibles a corto y medio plazo de Q, tal como pone de manifiesto la teoría austriaca del ciclo económico (Huerta de Soto, 2011). En concreto, el aumento de los medios de pago originado en un crecimiento del crédito no respaldado por ahorro real permitirá, a corto plazo, contratar a factores 
productivos que hasta el momento se encontraban desempleados por exigir una remuneración superior a su productividad marginal descontada. En la medida en que el crédito fiduciario genera aumentos insostenibles de la demanda de ciertas industrias y reducciones en los tipos de interés, la productividad marginal descontada calculada por muchos empresarios para ciertos factores aumentará, lo que les llevará a incorporarlos a sus planes de negocio y a incrementar con ellos la producción y el número de transacciones a corto plazo.

Sin embargo, como la composición de la estructura productiva se modificará por acción del crédito fiduciario, sobredimensionando ciertos sectores económicos a costa de no mantener o aumentar proporcionalmente otros, lo que significará que a medio plazo esa estructura deberá reajustarse y reconocer que la cantidad de bienes futuros que planificaba alumbrar será sustancialmente menor a la esperada. Este empobrecimiento generalizado propio de las crisis económicas hace que la cantidad de transacciones sea menor de la que habría sido sin el aumento inicial del crédito fiduciario.

Por consiguiente, los aumentos de $\mathrm{M}$ basados en la inflación de crédito fiduciario darán lugar a aumentos transitorios de $Q$ que en algún momento revertirán en forma de una cantidad de transacciones menor que sin la hinchazón inicial. Además, este proceso podría llegar a realimentarse con el ya comentado de las hiperinflaciones e hiperdeflaciones (según cuál sea la respuesta de las autoridades monetarias a la crisis).

En definitiva, no existe independencia ni cuantitativa ni cualitativa entre $\mathrm{M}$ y Q: las variaciones del primero afectarán al volumen y a la composición del segundo en una dirección sostenible o insostenible, según la naturaleza de la variación de M. De ahí que si bien la teoría cuantitativa ha resultado de gran importancia para alertar contra las consecuencias inflacionistas del aumento de $M$, también ha servido, al presuponer esa independencia entre $\mathrm{M}$ y $\mathrm{Q}$, para desviar la atención de otra consecuencia más dañina si cabe, como es la deformación en el volumen y la sostenibilidad de las transacciones. No fueron pocos los defensores de la teoría cuantitativa que, como Irving Fisher, sostuvieron que convenía estabilizar los precios ante los aumentos de $Q$ 
derivados de mejoras en la productividad porque, de este modo, se facilitaba el cálculo económico y no se generaba perjuicio alguno dentro del sistema económico (Huerta de Soto 2009, 383). Un error en gran medida responsable de la Gran Depresión y de la Gran Recesión.

IV

\section{CETERIS PARIBUS, LOS CAMBIOS EN LA OFERTA MONETARIA PROVOCAN VARIACIONES MECANICISTAS EN TODOS LOS PRECIOS}

La última de las hipótesis en las que se basa la teoría cuantitativa es la de que los aumentos en $\mathrm{M}$ dan lugar a incrementos automáticos y mecanicistas en los precios, con independencia de cómo se haya originado y extendido ese incremento en $\mathrm{M}$. La proposición puede tener dos significados: uno más restrictivo y otro más flexible. El primero se correspondería con sostener que todos los precios particulares se mueven en idéntica proporción a la variación de $\mathrm{M}$; el segundo, que $\mathrm{P}$ (el nivel agregado de precios; una media de todos los precios de la economía) aumenta o disminuye en la misma proporción que lo hace $M$, lo que permite que haya precios particulares que suban o bajen siempre que la variación de la media de todos ellos sea proporcional a la de M.

La primera de estas dos interpretaciones, que coincide con la célebre hipótesis de la neutralidad del dinero, es indudablemente falsa. Como ya descubriera Richard Cantillon - de ahí el famoso Efecto Cantillon-, las variaciones en los precios particulares de la economía dependen de los circuitos por los que se introduzcan los nuevos medios de pago. Con independencia de que el aumento de $\mathrm{M}$ proceda de un aumento de dinero $\left(\mathrm{M}^{\prime}\right)$ o de promesas de pago $\left(\mathrm{M}^{\prime \prime}\right)$, lo cierto es que los precios que con mayor intensidad y rapidez se incrementarán serán los de aquellos bienes en los que primero se gasten los nuevos medios de pago.

$Y$ dado que al comienzo esos nuevos medios de pagos se gastarán antes de que el conjunto de precios de la economía haya aumentado, todos los agentes económicos se verán forzados o incentivados a modificar sus patrones de gasto y con ellos los 
patrones de producción dentro de la economía. Es decir, aunque podría resultar verosímil la afirmación de que si todos los precios (incluidas las rentas) aumentaran a la vez un $10 \%$ no se producirían cambios en los patrones de gasto y producción de la economía, en el caso de que únicamente la renta monetaria de un solo agente aumentara un $10 \%$, parece claro que sí acaecerán redistribuciones en la disposición y producción de los bienes y servicios de una economía.

En definitiva, los aumentos de $\mathrm{M}$ no pueden traducirse en incrementos equiproporcionales de todos los precios por el simple hecho de que el dinero y las promesas de pago entran en la economía a través de ciertos circuitos (el gasto de los productores de dinero o el gasto de quienes generan promesas de pago) y es a través de esos circuitos por donde se va extendiendo la inflación de precios.

Nos queda, sin embargo, valorar el realismo de la interpretación flexible de esta cuarta hipótesis de la teoría cuantitativa, a saber, que la variación de la media de los precios es proporcional a la variación de la cantidad de medios de pago. En principio, de manera aislada nada tenemos que objetar contra la misma, pues si el resto de erróneas hipótesis de la teoría cuantitativa se asumen ciertas, la interpretación flexible de esta cuarta hipótesis deviene cierta por una necesidad lógica y matemática. Es decir, si V y $Q$ no varían ante cambios en $\mathrm{M}$ (esto es, si todos los medios de pago se emplean para adquirir la misma cantidad de bienes y servicios en idéntico lapso de tiempo), por fuerza la variación en la media de los precios ha de ser proporcional a la variación en la cantidad de medios de pago. Es, como ya hemos venido diciendo, una simple identidad contable que tan sólo iguala el importe de los pagos efectuados $\left(\mathrm{M}^{*} \mathrm{~V}\right.$, siendo en este caso $\mathrm{V}$ constante) con los cobros recibidos $\left(\mathrm{P}^{*} \mathrm{Q}\right.$, siendo en este caso $\mathrm{Q}$ constante).

Por ejemplo, si en una economía donde cada año (V) se gastan 1.000 onzas de oro $(\mathrm{M})$ en comprar un televisor y un automóvil (Q) que cuestan 200 y 800 onzas respectivamente (P), aumenta la cantidad de medios de pago a 2.000 onzas de oro y los patrones de gasto se modifican de tal forma que esa economía pasa a gastar 1.500 onzas en comprar un televisor y 500 en comprar un 
automóvil, tanto el índice Paasche como el índice Laspayres de precios se incrementarán en un $100 \%$, que es justo lo que ha aumentado M (de hecho, si Q no varía ambos índices son iguales), por mucho que el precio de los televisores haya aumentado y el de los automóviles haya caído. Estamos ante una simple identidad: si ahora gastamos 2.000 onzas en comprar los mismos bienes que antes, la suma de los precios de todos ellos será igual a 2.000 onzas (justo el doble que cuando gastábamos en esos mismos bienes 1.000 onzas).

Otra cuestión es la relevancia que esta conclusión merezca dentro del análisis económico. Al cabo, lo que guía las decisiones individuales no es el nivel agregado de precios, sino los precios relativos y, como vemos, los precios relativos sí pueden variar, originando cambios a medio plazo en $Q$ y por tanto volviendo inaplicable la teoría cuantitativa como marco teórico para una economía dinámica.

\section{$\mathrm{V}$ CONCLUSIÓN}

Ninguna de las cuatro hipótesis sobre las que, según Joseph Schumpeter, se asienta la versión canónica de la teoría cuantitativa son realistas; o, como mucho, sólo lo son en ciertas situaciones muy concretas. Siendo generosos, podemos concluir que la teoría cuantitativa será casi siempre falsa y sólo en algunas ocasiones - cuando casualmente coincidan las cuatro hipótesis como en una conjunción astral- la realidad se acercará a la predicción teórica. En la mayoría de los casos, sobre todo si pretendemos analizar largos períodos de tiempo a lo largo de los cuales se alternan numerosas variaciones cíclicas, la teoría cuantitativa es inservible.

Pero, como hemos afirmado al inicio, no habría que confundir la teoría cuantitativa con la ecuación cuantitativa. Lo segundo, en realidad, es simplemente una identidad contable en la que tiene cabida no sólo la teoría cuantitativa sino cualquier tipo de operación monetaria que podamos concebir. Al cabo, si relajamos todas las hipótesis de la teoría cuantitativa llegaremos a una cáscara hueca, sin contenido teórico alguno, que será precisamente 
la ecuación cuantitativa. Confundir ambas sería tanto como igualar un marco analítico con uno de los pésimos análisis que se desarrolla en su interior.

\section{REFERENCIAS BIBLIOGRÁFICAS}

Anderson, Benjamin (1917): The Value of Money. The Macmillan Company.

HAYEK, Friedrich (1967): Prices and Production. Augustus M. Kelly, Publishers.

Huerta De Soto, Jesús (2011): Dinero, crédito bancario y ciclos económicos. Unión Editorial, 5. a edición. Primera edición de 1998. Marget, Arthur (1938): The Theory of Prices. Prentice-Hall. Schumpeter, Joseph (1954): History of Economic Analysis. Oxford University Press. 